Case Report

\title{
An Unusual Initial Presentation of Lupus Nephritis as a Renal Mass
}

\author{
Remi Goupil, ${ }^{1}$ Annie-Claire Nadeau-Fredette, ${ }^{1}$ Virginie Royal, ${ }^{2}$ \\ Alexandre Dugas, ${ }^{3}$ and Jean-Philippe Lafrance ${ }^{1,4,5}$ \\ ${ }^{1}$ Nephrology Division, Hôpital Maisonneuve-Rosemont, Montreal, QC, Canada H1T 2M4 \\ ${ }^{2}$ Pathology Department, Hôpital Maisonneuve-Rosemont, Montreal, QC, Canada H1T 2M4 \\ ${ }^{3}$ Radiology Division, Hôpital Maisonneuve-Rosemont, Montreal, QC, Canada H1T 2M4 \\ ${ }^{4}$ Medicine Department, Universite de Montreal, Montreal, QC, Canada H3T $1 J 4$ \\ ${ }^{5}$ Centre de Recherche Hôpital Maisonneuve-Rosemont, Montreal, QC, Canada HIT 2M4 \\ Correspondence should be addressed to Jean-Philippe Lafrance; jean-philippe.lafrance@umontreal.ca
}

Received 29 August 2014; Revised 22 December 2014; Accepted 24 December 2014

Academic Editor: Kouichi Hirayama

Copyright (C 2015 Remi Goupil et al. This is an open access article distributed under the Creative Commons Attribution License, which permits unrestricted use, distribution, and reproduction in any medium, provided the original work is properly cited.

\begin{abstract}
Lupus nephritis is a frequent manifestation of systemic lupus erythematous. Lupus nephritis usually presents with abnormal urinalysis, proteinuria, and/or renal insufficiency. We report a case of a 48-year-old woman who underwent partial nephrectomy for a fortuitously discovered solid enhancing left kidney mass. No neoplastic cells were found in the biopsy specimen; however, the pathology findings were compatible with immune complex glomerulonephritis with a predominantly membranous distribution, a pattern suggestive of lupus nephritis. The mass effect was apparently due to a dense interstitial lymphocytic infiltrate resulting in a pseudotumor. Further investigation revealed microscopic hematuria with a normal kidney function and no significant proteinuria. Antinuclear antibodies were negative, although anti-DNA and anti-SSA/Rho antibodies were positive. A diagnosis of probable silent lupus nephritis was made and the patient was followed up without immunosuppressive treatment. After two years of follow-up, she did not progress to overt disease. To our knowledge, this represents the first case of lupus nephritis with an initial presentation as a renal mass.
\end{abstract}

\section{Introduction}

Lupus nephritis (LN) carries one of the highest morbidity and mortality risks of systemic lupus erythematous (SLE). Usually, LN will be suspected in the presence of active urine sediment, proteinuria, and/or renal insufficiency and confirmed by kidney biopsy. This report describes an unusual case of LN diagnosed fortuitously after a partial nephrectomy for a solid renal lesion.

\section{Case Presentation}

A 48-year-old woman with abdominal pain was diagnosed with acute cholecystitis based on findings on a computed tomography (CT) scan and ultrasound. The CT scan also showed a $15 \times 17 \mathrm{~mm}$ homogeneous, enhancing left renal lesion without fat or calcium contents (Figure 1(a)). Magnetic resonance imaging (MRI) revealed a focal left renal iso/mildly hypointense $\mathrm{T} 1$ and $\mathrm{T} 2$ lesion, with diffusion restriction, and a mild to moderate homogeneous enhancement, without fat content (Figures 1(b) and 1(c)). The differential diagnosis included papillary neoplasm of the kidney, focal lymphomatous infiltration, focal pyelonephritis, and pseudotumoral sarcoidosis. The patient underwent a partial left nephrectomy for a neoplasm suspicion. Pathology evaluation did not show any neoplastic cells but rather a focal and dense lymphocytic infiltrate resulting in a pseudotumor (Figure 1(d)). This interstitial infiltrate was predominantly composed of mature T lymphocyte, with fewer B lymphocytes, and rare plasmocytes, without IgG4 overexpression. Light microscopic evaluation of the glomeruli showed features of membranous glomerulonephritis with diffusely thickened glomerular basal membranes (GBM). Jones' silver stain revealed vacuolisation and spike appearance of the GBM. Few glomeruli showed 


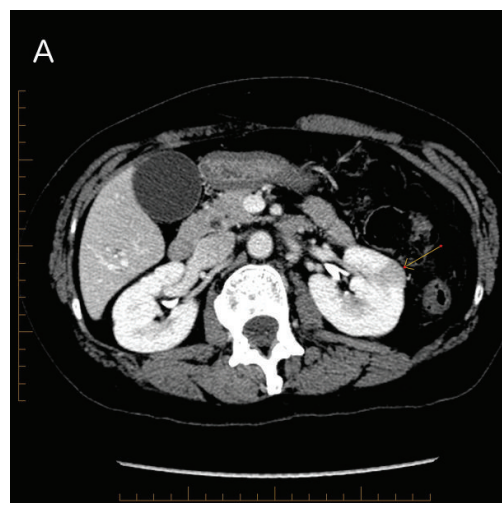

(a)

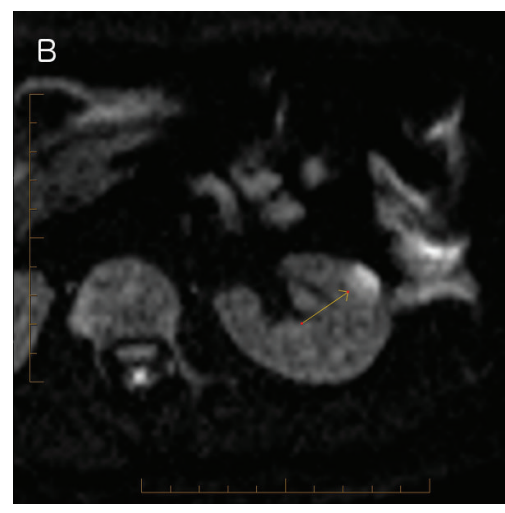

(b)

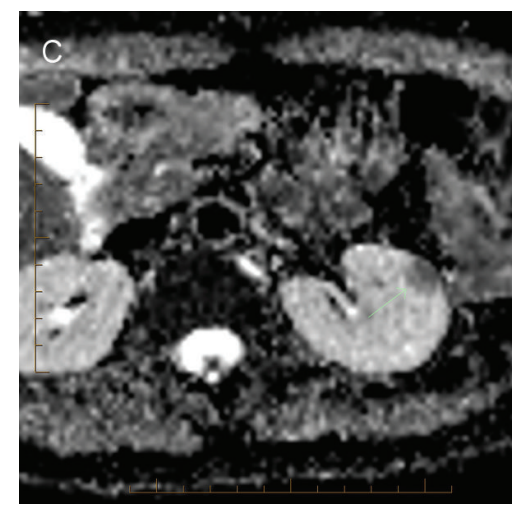

(c)

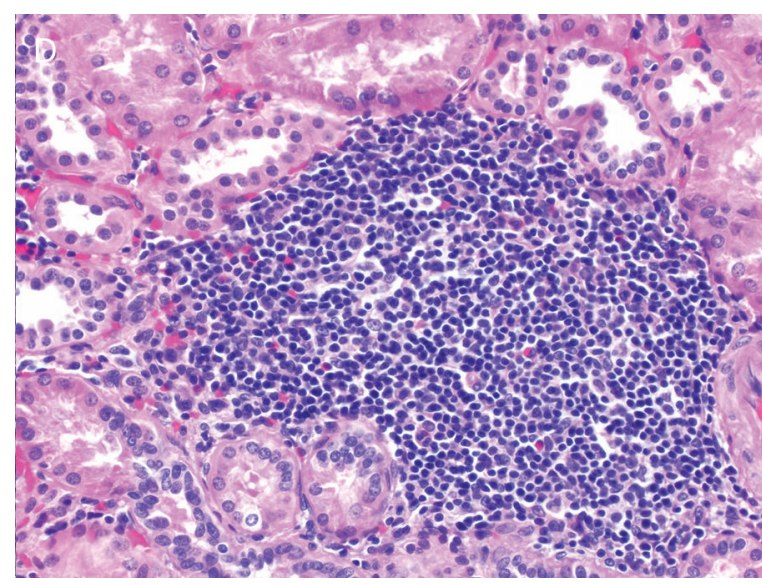

(d)

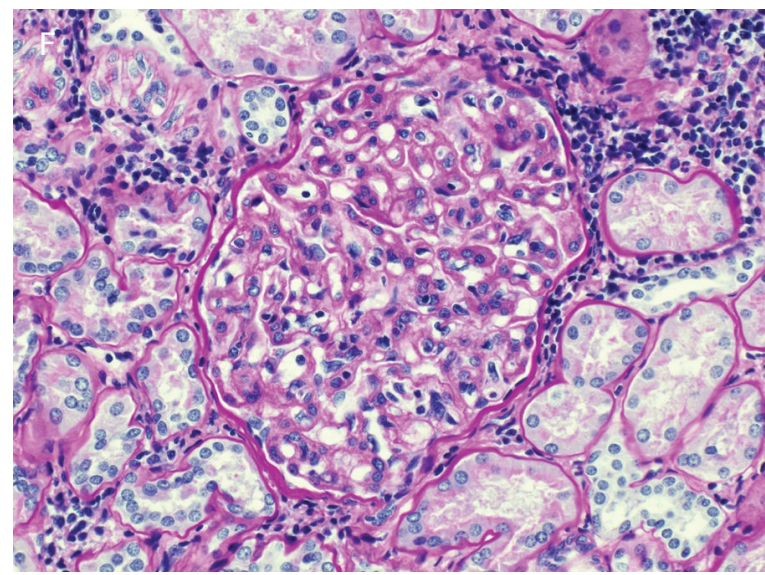

(f)

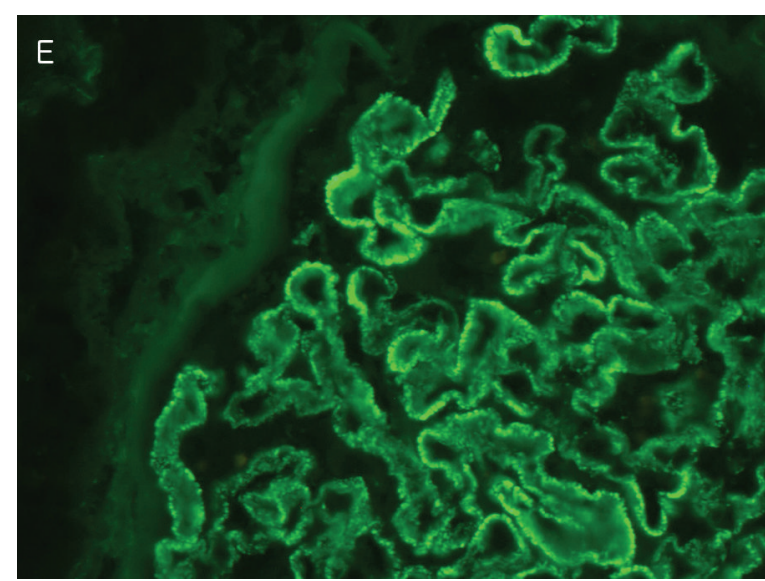

(e)

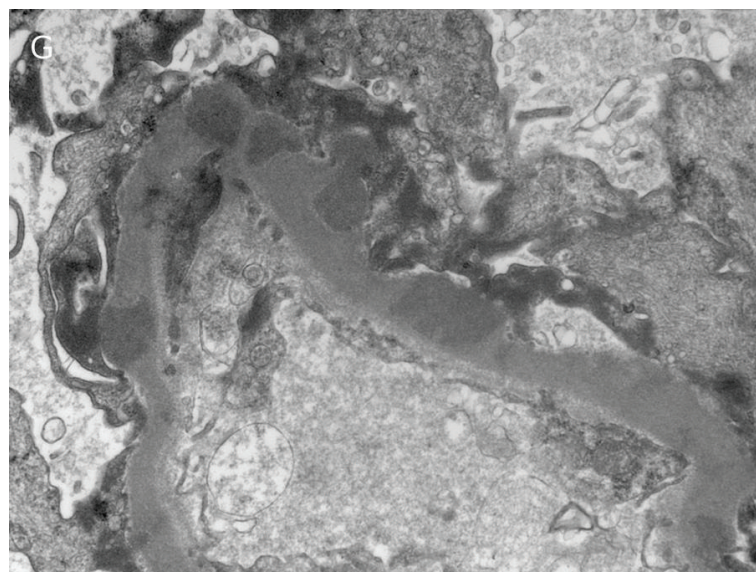

(g)

Figure 1: Radiologic and pathologic presentation of the renal lesion. (a) CT scan image showing a homogeneous, enhancing left renal lesion. (b) Diffusion renal magnetic resonance imaging (MRI) image of the same left renal lesion. (c) Apparent diffusion coefficient (ADC) MRI image. (d) Light microscopy with periodic acid-Schiff staining showing a lymphocytic infiltration. (e) Granular positivity of Clq on the glomerular basement membrane by immunofluorescence. (f) Higher magnification of endocapillary hypercellularity. (g) Electronic microscopy showing subepithelial deposits on the glomerular basal membrane.

a focal endocapillary hypercellularity and two presented fibrocellular crescents. Necrosis was not seen. Immunofluorescence microscopy showed granular positivity along the GBM and mesangium for $\operatorname{IgG}(3+$, on $0-3$ scale), $\operatorname{IgA}(3+)$, IgM (1+), C3 (3+), Clq (2+), Kappa (3+), and Lambda (3+)
(Figure 1(e)). Testing for IgG subclasses was uninterpretable, and testing for anti-PLA2R was not performed. Electron microscopy showed numerous subepithelial and mesangial deposits and rare small subendothelial deposits (Figure $1(\mathrm{~g})$ ). There were no extraglomerular deposits, and tubuloreticular 
TABLE 1: Investigation results at time of partial nephrectomy specimen and subsequent kidney biopsy after 12 months of clinical follow-up.

\begin{tabular}{lcc}
\hline \multirow{2}{*}{ Investigation } & \multicolumn{2}{c}{ Results } \\
& nephrectomy & Biopsy \\
\hline Serum creatinine & $60 \mu \mathrm{mol} / \mathrm{L}$ & $61 \mu \mathrm{mol} / \mathrm{L}$ \\
Microscopic hematuria & $3-5$ red blood & $3-5$ red blood \\
cells per field & cells per field \\
Urinary albumin/creatinine & $7.6 \mathrm{mg} / \mathrm{mmol}$ & $10 \mathrm{mg} / \mathrm{mmol}$ \\
ratio & $<1: 80$ & $<1: 80$ \\
ANA titer & $424 \times 10^{3} \mathrm{IU} / \mathrm{L}$ & $<55 \times 10^{3} \mathrm{IU} / \mathrm{L}$ \\
Anti-DNA & $10.4 \mathrm{IU} / \mathrm{L}$ & - \\
Anti-SSA/Rho & $15.9 \mathrm{IU} / \mathrm{L}$ & - \\
Anti-SSB/La & $1.22 \mathrm{~g} / \mathrm{L}$ & $1.08 \mathrm{~g} / \mathrm{L}$ \\
C3 complement & $0.15 \mathrm{~g} / \mathrm{L}$ & $0.19 \mathrm{~g} / \mathrm{L}$ \\
C4 complement & $28.7 \mathrm{~g} / \mathrm{L}$ & - \\
Serum IgG & $0.6 \mathrm{~g} / \mathrm{L}$ & - \\
IgG4 subclass & &
\end{tabular}

inclusions were not seen. Thus, a diagnosis of immune complex glomerulonephritis with a predominant membranous distribution was made, a pattern suggestive of lupus nephritis [1].

On further evaluation, the patient denied any symptoms which could have been related to SLE and physical examination was noncontributory. Past personal and familial medical history were noncontributory, without known autoimmune or renal conditions. Laboratory tests were as follows (Table 1): creatinine $60 \mu \mathrm{mol} / \mathrm{L}$, microscopic hematuria (3-5 red blood cells (RBC) per field on multiple occasions and 6-10 RBC per field once), absence of urinary casts, urine albuminto-creatinine ratio $7.6 \mathrm{mg} / \mathrm{mmol}$ (normal $<3.4$ ), negative antinuclear antibodies (ANA), anti-DNA $424 \times 10^{3} \mathrm{IU} / \mathrm{L}$ (normal $<55 \times 10^{3}$ ), anti-SSA/Rho and anti-SSB/La 10.4 and 15.9 IU/L, respectively (normal < 8.0), normal C3, lower limit of normal C4, serum IgG $28.7 \mathrm{~g} / \mathrm{L}$ (normal 5.5-16.3), IgG4 subclass $0.6 \mathrm{~g} / \mathrm{L}$ (normal 0.07-0.88), and negative HIV, hepatitis $\mathrm{B}$ and $\mathrm{C}$ viruses testing.

Considering the absence of major signs of disease activity, the patient was first observed without treatment. During the subsequent 12 months, the patient remained asymptomatic with persistent microscopic hematuria and microalbuminuria (Table 1). In order to clarify prognostic and to determine treatment indication, a right kidney biopsy was performed (11 glomeruli were obtained). The pathology confirmed an immune complex glomerulonephritis with a predominantly membranous distribution, with immunofixation showing deposition of IgG, IgA, IgM, C3, and C1q, but without any signs of focal proliferation. The patient was further observed without immunosuppressive therapy. Eighteen months after initial diagnosis, she reported arthralgia in both hands and was referred to a rheumatologist. She had no physical signs of lupus-related arthritis and, as hematuria and albuminuria had disappeared and both ANA and anti-DNA levels were negative, it was decided to continue observation without treatment.

\section{Discussion}

To our knowledge, this is the first description of a lupus nephritis diagnosed in the context of a renal lesion suggestive of malignancy. In the partial nephrectomy specimen, no tumorous or neoplastic cells were found but only features compatible with LN. IgG4 renal disease was considered as it may also present as inflammatory mass and membranous nephropathy, but IgG4 serum levels and immunostaining on the biopsy were not in favour of this diagnosis [2]. Renal disease in SLE is usually not associated with renal masses but, in this case, it appears it was the result of a dense lymphocytic infiltrate.

According to the Systemic Lupus International Collaborating Clinics (SLICC) group, biopsy-proven $\mathrm{LN}$ in presence of ANA or anti-DNA antibodies is now sufficient to make a diagnosis of SLE [3]. Renal involvement is frequent among SLE patients and presentation is highly variable [4]. True prevalence of renal-limited SLE is not well described, as this disease is usually accompanied by systemic manifestations on diagnosis or can herald the arrival of clinical SLE. However, cases of LN with negative serologies and without subsequent development of systemic involvement have been described [5].

Silent LN is usually defined as biopsy-proven $\mathrm{LN}$ without any renal clinical evidence of disease activity [6]. In silent LN, renal pathology usually shows isolated mesangial disease, although it can also demonstrate focal of diffuse proliferative disease. In a series of 86 SLE patients without clinical signs of renal involvement, 15 percent had class III or IV nephritis and 10 percent had class V membranous nephritis [7]. Prognosis of silent LN ranges from benign renal evolution to progression into a clinically active disease $[7,8]$.

This case of lupus nephritis is unusual because of its initial manifestation as a malignancy-suspected renal mass. The evolution suggests a case of early silent LN. Prevalence, prognosis, and usefulness of immunosuppressive treatment in similar cases remain unclear as this condition would usually not warrant a kidney biopsy. Further studies are needed to better characterize silent LN.

\section{Key Message}

Lupus nephritis can present itself as a solid mass suggestive of neoplasm.

\section{Ethical Approval}

Publication of this case report was approved by the Director of the professional services at Maisonneuve-Rosemont Hospital.

\section{Consent}

The patient described in the case report had given informed consent for the case report to be published. 


\section{Conflict of Interests}

The authors have nothing to declare.

\section{References}

[1] J. J. Weening, V. D. D’Agati, M. M. Schwartz et al., "The classification of glomerulonephritis in systemic lupus erythematosus revisited," Kidney International, vol. 65, pp. 521-530, 2004.

[2] L. D. Cornell, "IgG4-related kidney disease," Current Opinion in Nephrology and Hypertension, vol. 21, pp. 279-288, 2012.

[3] M. Petri, A. M. Orbai, G. S. Alarcón et al., "Derivation and validation of the Systemic Lupus International Collaborating Clinics classification criteria for systemic lupus erythematosus," Arthritis \& Rheumatology, vol. 64, no. 8, pp. 2677-2686, 2012.

[4] N. Kasitanon, L. S. Magder, and M. Petri, "Predictors of survival in systemic lupus erythematosus," Medicine, vol. 85, no. 3, pp. 147-156, 2006.

[5] A. Huerta, A. S. Bomback, V. Liakopoulos et al., "Renal-limited 'lupus-like' nephritis," Nephrology Dialysis Transplantation, vol. 27, no. 6, pp. 2337-2342, 2012.

[6] M. E. Zabaleta-Lanz, L. E. Muñoz, F. J. Tapanes et al., "Further description of early clinically silent lupus nephritis," Lupus, vol. 15, no. 12, pp. 845-851, 2006.

[7] D. Wakasugi, T. Gono, Y. Kawaguchi et al., "Frequency of class III and IV nephritis in systemic lupus erythematosus without clinical renal involvement: an analysis of predictive measures," The Journal of Rheumatology, vol. 39, no. 1, pp. 79-85, 2012.

[8] M. R. Gonzalez-Crespo, J. I. Lopez-Fernandez, G. Usera, M. J. Poveda, and J. J. Gomez- Reino, "Outcome of silent lupus nephritis," Seminars in Arthritis and Rheumatism, vol. 26, no. 1, pp. 468-476, 1996. 


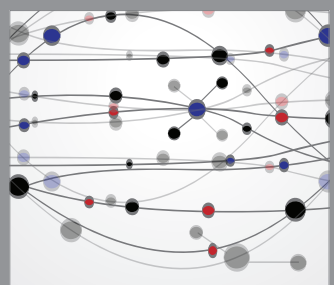

The Scientific World Journal
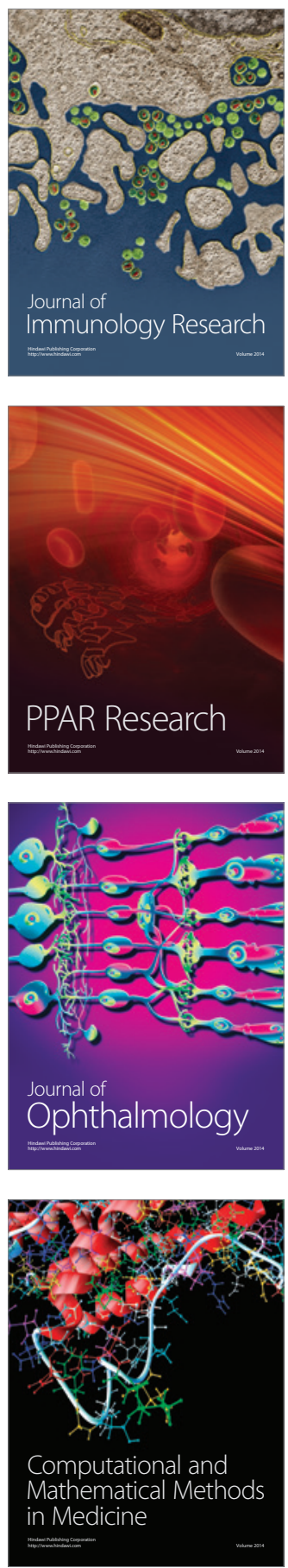

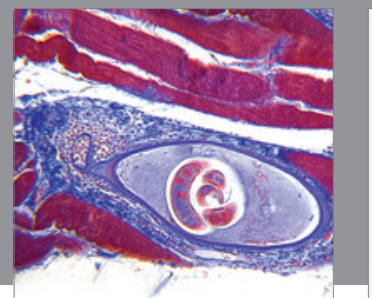

Gastroenterology

Research and Practice
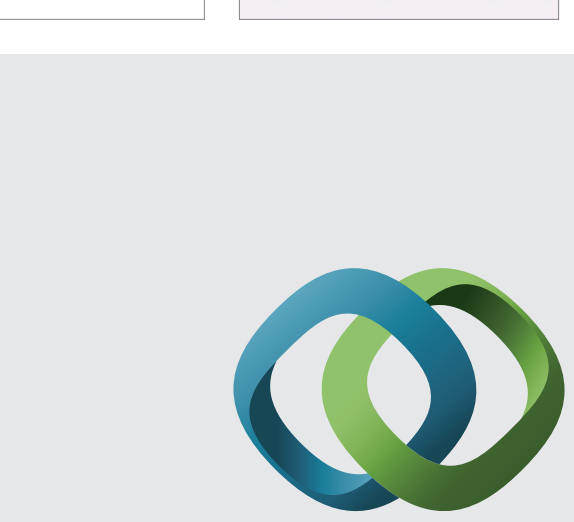

\section{Hindawi}

Submit your manuscripts at

http://www.hindawi.com
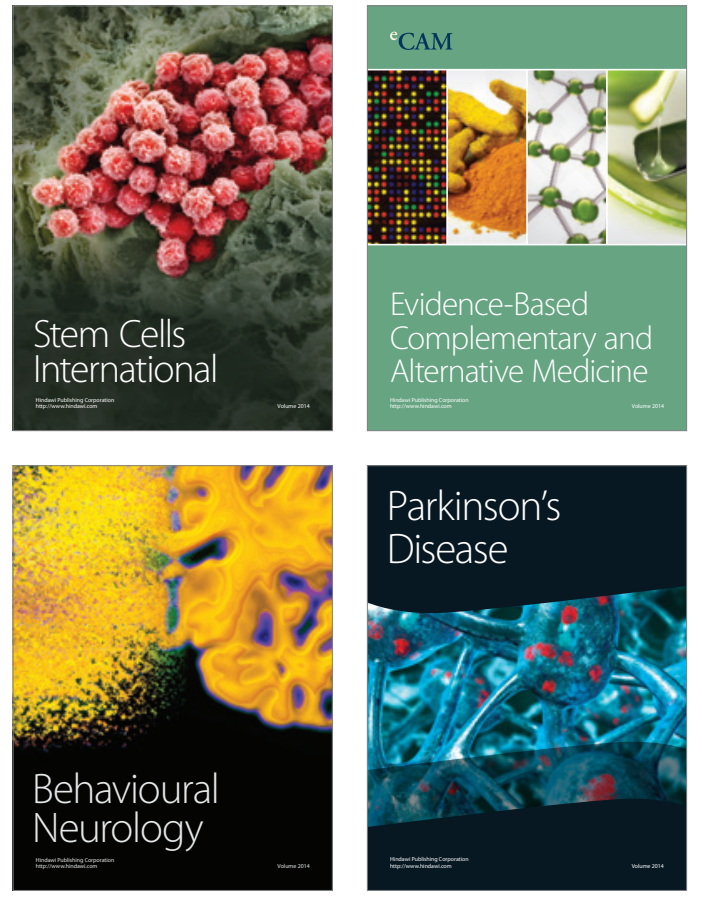
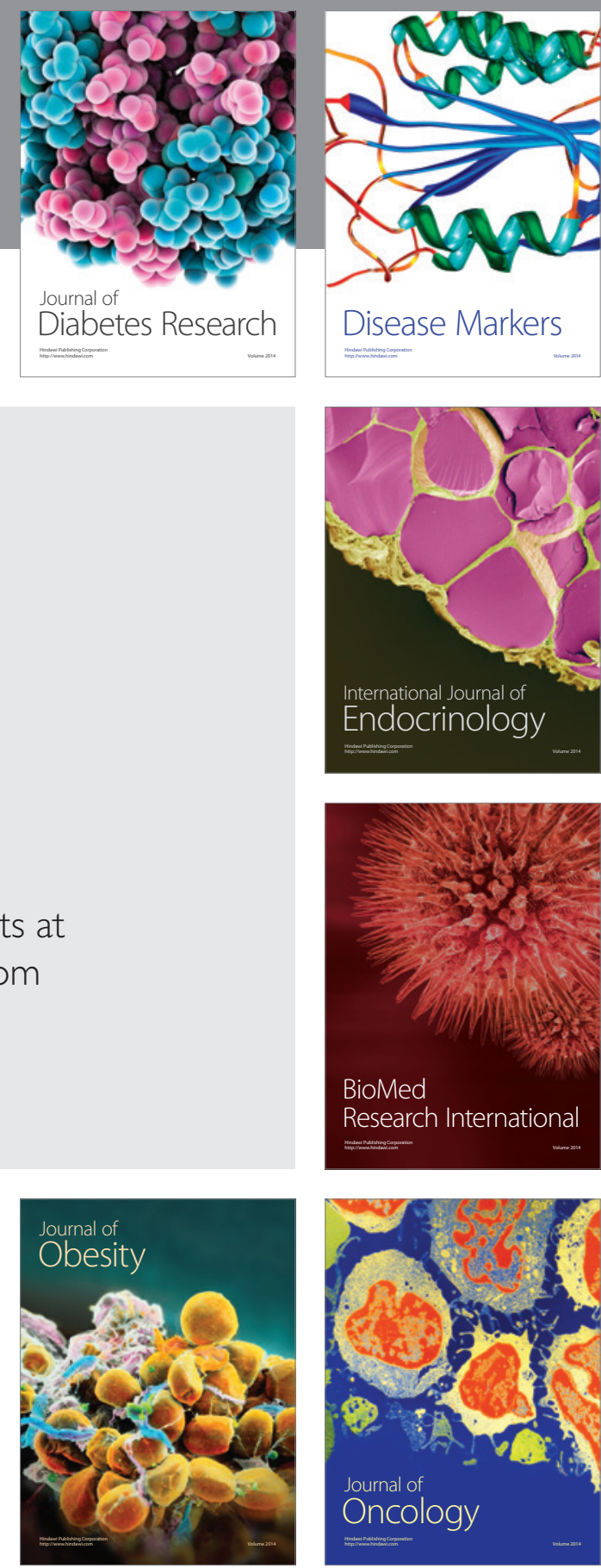

Disease Markers
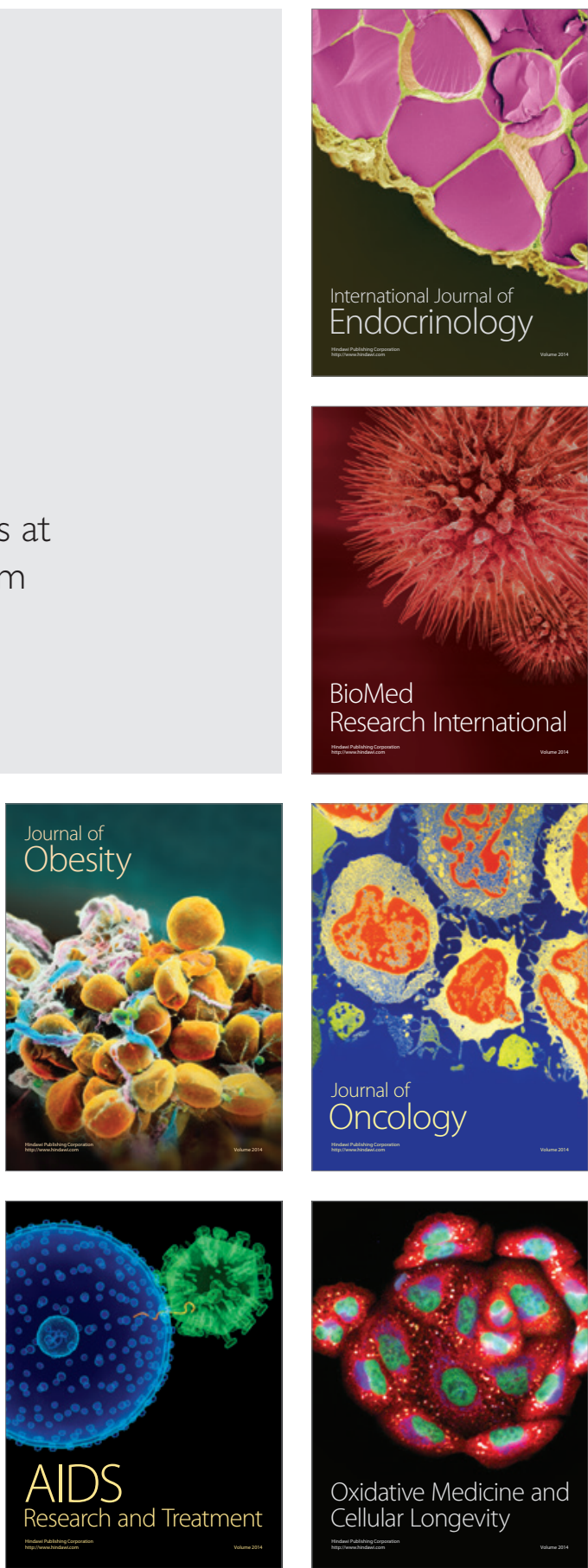